\title{
Oral cancer public policies: Is there any evidence of impact?§
}

\author{
Cassius Torres-Pereira ${ }^{(a)}$ \\ (a) $\mathrm{PhD}$ in Oral Medicine, Professor, \\ Stomatology Department, Federal University \\ of Paraná, Curitiba, PR, Brazil.
}

\begin{abstract}
Investigation in oral cancer comprises many different fields such as epidemiology, risk factors, biological markers, diagnostic testing, screening, treatment and prognosis. Although many researchers have dealt with the oral cancer problem, it is unknown if any public policy is capable of reducing its incidence around the world under the scrutiny of the scientific method. This paper aims to briefly review and discuss the literature regarding oral cancer public policies and to screen the evidence of controlled implementations of oral health policies that have been able to diminish oral cancer incidence around the globe.
\end{abstract}

Descriptors: Mouth Neoplasms; Public Health; Public Policy

\section{Introduction}

Oral cancer is a major problem in oral health. Late diagnosis, high mortality rates and morbidity leading to important disfigurement are characteristics of the disease worldwide. Many proposals of public health interventions are cited in the literature but little is known about an evidence-based approach capable of impacting oral cancer incidence or providing early diagnosis of the disease. Oral squamous cell carcinomas (OSCC) are responsible for $90-95 \%$ of the new cases of oral cancer. ${ }^{1}$ In order to critically evaluate public health policies aiming to control oral malignization, this paper will deal with the particularities of OSCC.

\section{Epidemiology of oral cancer: New trends?}

Oral cancer epidemiology has been extensively described in many countries. Incidence, prevalence and mortality rates have been the object of numerous studies and are well summarized elsewhere. ${ }^{1}$ Although oral cancer is not as common when compared to other human cancers like those affecting skin, breast, cervix or lung, it can be identified mainly in a very characteristic group of patients. Oral Squamous Cell Carcinomas (OSCC) share similar risk factors described for other head and neck malignancies like pharyngeal and maxillary sinus cancer. In some countries, researchers have identified a recent oral cancer trend of manifesting itself in young people, and also a recent incidence growth among women. ${ }^{2}$

The regional disparities in incidence and mortality rates were not only described among countries but within them. A study using data from the National Cancer Institute's Surveillance, Epidemiology and End Results (SEER) program in the United States revealed that 8 out of the 51 North American states exhibited increasing rates of oral cancer deaths despite 
the overall decrease in mortality over the past 30 years in that country. ${ }^{3}$ Authors claim that emphasis should be given to regional disparities when facing the problem of oral malignization, thus requiring a more careful approach to this aspect of the disease.

\section{Risk factors: What is different from the already known?}

Although many agents/behaviors have been linked to oral malignization, cumulative data have pointed out that tobacco consumption, in any of its presentations, and alcohol drinking are the strongest and most important predictors for intraoral malignant transformation. The risks for both tobacco and alcohol are described as very dose-dependant. Even in lip cancer, where occupational (or not) sun exposure could explain cases of malignancy, tobacco increases the number of cases of semi-mucosa malignant transformation..$^{1,2,3,4}$ Not only exposure to alcohol and tobacco but also habit cessation reinforces this strongest causal-effect association. Data reviewed from case-control studies in the International Head and Neck Cancer Epidemiology Consortium pooled 17 studies on smoking cessation (12,040 cases / 16,884 controls) and 13 studies on drinking cessation (9,167 cases / 12,593 controls). Researchers demonstrated a head and neck oral cancer incidence reduction that achieved the same risk of disease development of never smokers / drinkers after 20 years of habit quitting. ${ }^{5}$ Recently, attention has been focused on alcohol-containing mouthrinses. Although most information relies yet on biological plausibility, some authors call attention to mouthrinse adequate and proper indications. ${ }^{6}$ Some authors have reported that the effect of socioeconomic status on oral cancer is given little recognition in a predominantly medical model approach to research and prevention on the risks of the disease. A paper published in 2008 summarizes results from studies which had included this variable as an outcome or an independent predictor. ${ }^{7}$ Forty-one case-control studies including a total of 15,344 individuals with oral cancer and 33,852 controls were included. Compared with individuals who were in high socioeconomic strata, the pooled odds ratios (OR) for the risk of developing oral can- cer were 1.85 ( $n=37$ studies) for those with low educational attainment; $1.84(n=14)$ for those with low occupational social class; and $2.41(n=5)$ for those with low income. Socioeconomic status was significantly associated with an increased oral cancer risk regardless of whether a high- or low-income country was studied. In a study comparing health insured versus uninsured individuals in the United States, uninsured patients were significantly more likely to present with an advanced-stage head and neck malignancy at diagnosis, with an OR of 2.94, and to present with at least 1 positive lymph node $(\mathrm{OR}=1.84)$ compared with patients with private insurance. Patients uninsured were at increased risk of death after a diagnosis of head and neck SCC when compared with patients with private insurance. Researchers claim their results to be statistically adjusted for age, gender, race, smoking, alcohol use, site, socioeconomic status, treatment, and cancer stage. ${ }^{8}$ Oral-related quality of life is being frequently reported as a major concern for patients diagnosed and treated for mouth malignant neoplasms. Some studies strongly suggest that oral-related quality of life should be adopted as a standard criterion in hospital settings for the evaluation of oral cancer patients. ${ }^{9}$

\section{Oral cancer "campaigns", mouth self-examination and screening for oral cancer: any evidence of effectiveness? \\ Campaigns}

A study conducted in Scotland ${ }^{10}$ evaluated a mass-media TV campaign "to increase people's feelings of personal risk and perceived susceptibility while at the same time avoiding raising unnecessary anxiety and encouraging inappropriate self-referrals". Authors interviewed a representative sample of the target population in both the campaign area and controls outside of the target area. A survey was conducted before the campaign was launched, and at 7 and 12 months after. A total of 922, 934 and 944 completed interviews were respectively achieved at baseline and first and second follow-up. The results presented a short-term, but not a longterm impact on awareness of the disease and inten- 
tion to respond to the symptoms targeted by the campaign. A study was conducted to investigate if a social marketing media campaign could reduce oral cancer racial disparities. The authors surveyed a cohort of residents in an intervention city (250) and a control city (250) immediately prior to and after the campaign. Participants (125 African-American and 125 white) in each city completed surveys at baseline and follow-up. The intervention city residents showed an increase in recognition of the campaign, awareness of the oral cancer exam, and interest in getting an exam, while no significant differences were found for the control city. African-Americans in the intervention city were more likely than whites to show increases in awareness of the campaign, oral cancer awareness, and interest in receiving an oral cancer exam. ${ }^{11}$ In Australia, researchers have evaluated the intention to quit after exposure to tobacco graphic health warning TV campaigns. Dentists were also asked to evaluate if there was any increased demand for smoking cessation advice in dental practices. ${ }^{12}$ Health warning pictures seemed to increase the patients' awareness and intention to quit smoking; however, they did not appear to generate more demand for smoking cessation advice from their dentists. Dentists had low expectations about their patients' motivation to stop smoking. Brazilian researchers evaluated the number of people whose problem was resolved at the primary care level or in referral services and the number of persons with a confirmed diagnosis of oral cancer after an oral cancer campaign conducted in conjunction with the annual flu vaccination campaign for the elderly in the state of São Paulo, Brazil. ${ }^{13}$ Authors concluded that the oral cancer prevention and early diagnosis program was ineffective, given the lack of monitoring of results in most parts of the state of São Paulo, and the high proportion of patients whose soft tissue lesion were not resolved. The usefulness of continuing the program in future years was questioned. The conclusion was based on results showing that from the people who were referred for diagnostic investigation of soft tissue lesions, $60.5 \%$ had their problem resolved, $0.5 \%$ (26 cases) had a confirmed diagnosis of oral cancer, and $22.5 \%$ did not have the diagnostic investigation completed. For $16.5 \%$ of the cases referred for further study, there was no information available concerning follow-up and outcomes.

\section{Mouth self-examination (MSE)}

Many references suggest that MSE could be an integral part of health policies for oral cancer. The technique is described generally as a non-invasive, low-cost, reliable and mass-applicable method to control oral cancer incidence. Moreover, its benefits would be targeted to secondary prevention. The patient would be capable to identify suspicious lesions for malignancy and then refer him/herself to a health primary or secondary care facility. One pilot study published recently resulted in 33\% sensitivity and $54 \%$ specificity for MSE. The positive predictive value of MSE was $17 \%$, and the negative predictive value was $73 \%$ in a sample with $22 \%$ prevalence of potentially malignant lesions. ${ }^{14}$

\section{Screening}

The strategy to identify patients through massscreening programs has received increasing attention. The possibility to identify patients with potentially malignant lesions or at risk for oral cancer development was reviewed in a 2006 metanalysis. ${ }^{15}$ Researchers concluded that there was insufficient evidence to support or refute the use of visual examination as a method of screening for oral cancer in the general population. Adjunctive measures to visual screening such as the use of toluidine blue, fluorescence imaging or brush biopsy showed no benefits or harm. The American Dental Association (ADA) Council on Scientific Affairs convened a panel to evaluate the available evidence regarding oral cancer screening and the use of other adjuncts. ${ }^{16}$ The panelists recommended that an oral cancer community-based screening could only reduce mortality if targeted to people who use tobacco, alcohol or both. In asymptomatic or patients presenting potentially malignant lesions there is insufficient evidence to show impact in mortality.

\section{Diagnostic delay}

Analysis of the time elapsed from the first symptoms of OSCC to treatment administration shows 
that diagnostic delay can be responsible for higher rates of morbidity and mortality. In a review of the literature, the authors emphasized the need to recognize the agents responsible for diagnostic delay, and that it could be attributable to the patient, the healthcare provider and the health system. ${ }^{17}$ They claim the need for methodologically adequate investigations that consider cultural and geographical aspects and use patient survival as the final outcome. In Brazil, authors have studied the time elapsed between the first symptoms, diagnosis and treatment of oral cancer patients. ${ }^{18}$ Major study endpoints comprised evolution time, time elapsed from referral to hospital and first appointment, time elapsed from first appointment and treatment, total time elapsed from first noticing the lesion and starting treatment. The results presented showed delays occurring in every step of the diagnosis and referral needed until the start of treatment. In some cases, this delay was up to 263 days.

\section{Discussion}

Even considering local disparities and new evidence of minor shifts in head and neck incidence numbers, it is well established that oral squamous cell carcinoma (OSCC) has a male predilection and presents manifestations mainly in adults over 40 years of age. ${ }^{1-3}$ Research about oral cancer risks are numerous and include meta-analysis, systematic reviews, longitudinal, transversal and case-control studies. Although a variety of suspected risk factors have been proposed as causal or co-factors in OSCC development, ${ }^{1,4-8}$ strategies with real impact on health policies should emphasize restriction or elimination of tobacco and alcohol consumption.

Many factors can be blamed for the current situation of late-stage oral cancer diagnosis, high morbidity and mortality around the world. The differences observed in epidemiological data from distinct global regions seem to have a greater importance for socioeconomic explanatory variables than the most researched biomedical models in explaining why there are so few advances in lowering incidence and providing earlier diagnosis for patients suffering from this disease. ${ }^{4-9}$ Conway et al. (2008) stated that "efforts to reduce exposure to risk factors alone are unlikely to succeed unless they are supported by measures designed to improve socioeconomic circumstances and to reduce socioeconomic inequalities. Health professionals and policy makers need to consider advocating for socioeconomic change in addition to behavior change."

It is frequently proposed that mass-media or more targeted campaigns and mouth self-examination could be of benefit as public strategies for the oral cancer problem. ${ }^{10-14}$ Although simple and very attractive, these methods have seldom been scientifically validated as oral cancer health strategies. The paucity of references dealing with the issue under the rigor of science is impressive and is in clear disagreement with the enthusiasm that often characterizes governments, specialty societies and other organizations in implementing them. On the other hand, the lack of evidence does not mean strategies could not be effective, but they clearly need to be studied more carefully. From the public health policy standpoint, however, it seems that health promotion initiatives are much more complex than establishing episodic initiatives like TV or other mass-media campaigns. ${ }^{10-12}$ Research efforts on the socioeconomic and behavioral impacts of public health policies and on the oral health care network organization are much less frequent than those on biological or treatment protocols. ${ }^{7}$

An interesting and growing discussion in many studies is the concept of mass screening. This technique could benefit from a "population-at-risk" approach. Searching for patients exposed to known and well established risk factors seems to be a more predictable strategy than searching for oral cancer at random or in an individual model of health care. ${ }^{15,16}$ Although evidence shows that oral cancers can be identified in a mass-screening initiative, the health policies' cost-effectiveness to support oral cancer screening should not rely on the basis of a few identified individual cases, but rather on the impact of the organized health network and epidemiological results of this strategy over time. ${ }^{15} \mathrm{~A}$ common approach is to confound what would be expected from an individual model of health care to be proposed as a general and wide public health policy. Mass-media approaches and oral cancer secondary preven- 
tion should be targeted to those at most risk of oral cancer. ${ }^{4-17}$ These individuals are described as very unlikely to become regular dental attendees. ${ }^{14}$

Diagnostic delay is a major concern in oral cancer. An expected increase in morbidity and survival rates is demonstrated when patients are not aware of or minimize the importance of seeking primary care upon the onset of symptoms. But delay can be also, in some cases, attributable to the health professional in cases of misdiagnosis or wrong and late referrals. ${ }^{17,18}$ Moreover, even when correctly diagnosed and referred, patients could expect delay to occur in the tertiary health care due to other referral issues, lack of a sufficient number of specialists, laboratories or hospitals. If the aforementioned situation is well described and a relevant problem in developed nations, delay can be expected to be even more dramatic in low-income economies and impoverished areas. ${ }^{18}$

Oral cancer public policy is a complex issue that needs a comprehensive approach. ${ }^{4}$ Although there is an expressive amount of literature that allows us to clearly diagnose the dimensions of the oral cancer public health problem, there is a clear lack of re-

\section{References}

1. Warnakulasuriya S. Global epidemiology of oral and oropharyngeal cancer. Oral Oncol. 2009 Apr-May;45(4-5):30916.

2. Shiboski CH, Schmidt BL, Jordan RC. Tongue and tonsil carcinoma: increasing trends in the US population ages 20-44 years. Cancer. 2005 May;103(9):1843-9.

3. Kingsley K, O’Malley S, Ditmyer M, Chino M. Analysis of oral cancer epidemiology in the US reveals state-specific trends: implications for oral cancer prevention. BMC Public Health. 2008 Mar 10;8:87.

4. Petersen PE. Oral cancer prevention and control - the approach of the World Health Organization. Oral Oncol. 2009 AprMay;45(4-5):454-60.

5. Marron M, Boffetta P, Zhang ZF, Zaridze D, Wünsch-Filho V, Winn DM, et al. Cessation of alcohol drinking, tobacco smoking and the reversal of head and neck cancer risk. Int J Epidemiol. 2010 Feb;39(1):182-196.

6. Lemos-Junior CA, Villoria GE. Reviewed evidence about the safety of the daily use of alcohol-based mouthrinses. Braz Oral Res. 2008 Aug;22 Suppl 1:24-31.

7. Conway DI, Petticrew M, Marlborough H, Berthiller J, Hashibe M, Macpherson LM. Socioeconomic inequalities and oral search to provide solid evidence on strategies to establish realistic and long-term effective approaches with evidence-based impact on OSCC incidence.

\section{Conclusions}

Although the epidemiology, risk factors, early diagnosis strategies, treatment and even biological markers of oral cancer are the subject matter of intensive research efforts, little is known about the real impact of public interventions on preventing the disease or minimizing its morbidity. From a strict scientific approach, there is no evidence to support the claim that any public intervention could have a quantitative impact on oral cancer incidence and morbidity other than tobacco and alcohol consumption control. These factors should be emphasized as major targets for public policy aimed to positively impact the numbers of oral cancer. In addition, there seems to be a low recognition that reducing inequalities could lower oral cancer numbers. Government, scientific societies, researchers and clinicians should combine efforts in order to demand and produce more solid scientific evidence of impact when evaluating or proposing oral cancer public policies.

cancer risk: a systematic review and meta-analysis of casecontrol studies. Int J Cancer. 2008 Jun;122(12):2811-9.

8. Kwok J, Langevin SM, Argiris A, Grandis JR, Gooding WE, Taioli E. The impact of health insurance status on the survival of patients with head and neck cancer. Cancer. 2010 Jan;116(2):476-85.

9. Andrade FP, Ferreira JLA, Durazzo MD. Evaluation of the quality of life of patients with oral cancer in Brazil. Braz. Oral Res. 2006 Dec;20(4):290-6.

10. Eadie D, MacKintosh AM, MacAskill S, Brown A. Development and evaluation of an early detection intervention for mouth cancer using a mass media approach. Br J Cancer. 2009 Dec;101 Suppl 2:S73-9.

11. Watson JM, Tomar SL, Dodd V, Logan HL, Choi Y. Effectiveness of a social marketing media campaign to reduce oral cancer racial disparities. J Natl Med Assoc. 2009 Aug;101(8):77482.

12. Afifah R, Schwarz E. Patient demand for smoking cessation advice in dentist offices after introduction of graphic health warnings in Australia. Aust Dent J. 2008 Sep;53(3):208-16.

13. Antunes JL, Toporcov TN, Wünsch-Filho V. [The effectiveness of the oral cancer prevention and early diagnosis 
- Oral cancer public policies: Is there any evidence of impact?

program in São Paulo, Brazil]. Rev Panam Salud Publica. 2007 Jan;21(1):30-6. Portuguese

14. Scott SE, Rizvi K, Grunfeld EA, McGurk M. Pilot study to estimate the accuracy of mouth self-examination in an at-risk group. Head Neck. 2010 Feb 9. Epub ahead of print

15. Kujan O, Glenny AM, Oliver RJ, Thakker N, Sloan P. Screening programmes for the early detection and prevention of oral cancer. Cochrane Database Syst Rev. 2006 Jul;19(3): CD004150.

16. Rethman MP, Carpenter W, Cohen EE, Epstein J, Evans CA, Flaitz CM et al. Evidence-Based Clinical Recommendations
Regarding Screening for Oral Squamous Cell Carcinomas. J Am Dent Assoc. 2010 May;141(5):509-20.

17. Gómez I, Warnakulasuriya S, Varela-Centelles PI, LópezJornet P, Suárez-Cunqueiro M, Diz-Dios P, et al. Is early diagnosis of oral cancer a feasible objective? Who is to blame for diagnostic delay? Oral Dis. 2010 May;16(4):333-42.

18. Abdo EN, Garrocho A de A, Barbosa AA, Oliveira EL, Franca-Filho L, Negri SL, et al. Time elapsed between the first symptoms, diagnosis and treatment of oral cancer patients in Belo Horizonte, Brazil. Med Oral Patol Oral Cir Bucal. 2007 Nov;12(7):E469-73. 